\title{
Paradigm Shift in Management Strategies of Craniocerebral Missile Injuries Improving Survival Rates and Functional Outcome Score
}

\author{
Binoy Kumar Singh ${ }^{1}$ Shameem Ahmed ${ }^{1}$ Basanta Kumar Baishya ${ }^{1}$ Zakir Hussain ${ }^{1}$ Rajib Hazarika ${ }^{2}$ \\ Ashim Saikia²
}

${ }^{1}$ Department of Neurosurgery, Guwahati Medical College and Hospital, Bhangagarh, Guwahati, India

${ }^{2}$ Department of Anaesthesiology, Guwahati Medical College and Hospital, Bhangagarh, Guwahati, India

Address for correspondence Binoy Kumar Singh, MS, Department of Neurosurgery, Guwahati Medical College and Hospital, Bhangagarh, Guwahati, India (e-mail: drbinoysingh@yahoo.com).

Indian J Neurotrauma 2015;12:53-61.

\begin{abstract}
\section{Keywords}

- craniocerebral missile injury

- Glasgow Coma Scale

- intracranial pressure

- neurological deficit

Introduction With development of firearms, civilian violence, ethnic clashes, militancy, terrorist attack, and military operations there is a worldwide increase in incidence of craniocerebral missile injuries. The mortality of patients with craniocerebral missile injuries is high, and if the victim survives, functional outcome is poor. In spite of high mortality, no proper attention was given in last five decades in improving surgical techniques and resuscitation patterns of patients with craniocerebral missile injuries.

Problems Considered The problems are many, but the two problems highlighted are decreased survival because of the inadequate pre-op resuscitation and poor functional outcome due to aggressive surgery. Therefore, the study was performed to evaluate the outcome of early and less aggressive surgical strategy and aggressive preoperative resuscitation in patients with penetrating injuries and also to look for factors prognosticating outcome.

Methods A series of 96 patients with craniocerebral missile injuries to head were managed at a single center over a period of 3 years is presented. Aggressive resuscitation was done in all hemodynamically unstable patients and patients with coagulopathy either with blood products, hyperosmolar therapy or ionotropic support on protocols of damage control resuscitation. Blood product therapy consisted of packed red blood corpuscles, fresh frozen plasma (FFP) and platelets. Hypertonic saline was used for hyperosmolar resuscitation, and for ionotropic support dopamine, adrenalin, and vasopressin were used. Minimum debridement of the missile tract was done and maximum cortical tissue was preserved.

Results Neurosurgical procedures contemplated on 78 patients. Criteria for craniotomy were-Glasgow Coma Scale (GCS) of $<8$ without hypotension and reacting pupils, GCS $>8$ with intracranial bone fragments/significant clot, and raised intracranial pressure. Surgical approach were frontotemporal exposure $(41 \%$, $n=32 / 78)$, frontoparietal exposure $(22 \%, n=18 / 78)$, retrosigmoid exposure ( $2 \%$, $n=2 / 78)$, combined middle and posterior fossa approach $(2 \%, n=2 / 78)$, and
\end{abstract}


decompressive craniectomy $(43 \%, n=24 / 78)$. Procedures done were debridement of devitalized tissue $(100 \%, n=78 / 78)$, extraction of bone fragment $(43 \%, n=17 / 39)$, extraction of metallic pellet $(62 \%, n=42 / 67)$, lax duraplasty $(100 \%, n=78 / 789)$, debridement of missile tract $(100 \%, n=78 / 78)$. Overall, 36 patients were operated within 8 hours and 22 patients were operated within 14 hours. Median age group were 26 years, $81 \%$ were male, surgical mortality of $18 \%$, and overall mortality was $33 \%$.

Conclusion This study indicates the factors prognosticating outcomes and supports that less aggressive surgery and aggressive preoperative resuscitation has decreased surgical mortality rates and improved functional outcomes.

\section{Introduction}

Despite the worldwide increase in the incidence of ballistic injuries, there are few published series that has attempted to change the management techniques causing improved survival. Missile injuries to head comprises majority of penetrating brain injuries accounting approximately 35\% of deaths from brain injury in persons younger than 45 years of age and $90 \%$ of patients die before reaching the hospital (Source: US Statistics, eMedicine, Penetrating head trauma, 2009). The entry wound is small but causes devastating injury and high casualties. Every possible attempt is made to develop the fire arms and artillery worldwide since the discovery of gunpowder to establish the superiority and earn peace resulting in more serious injuries. Aggressive surgical management was advocated earlier even in hemodynamically unstable patients and with poor GCS status by trauma surgeons with high mortality rates. But in recent times, mortality has not increased compared with the increase in incidence of missile injuries because of the better prehospital care, rapid transport, improved resuscitation techniques, and early surgery of such victims. The concept of early and definitive surgery with debridement of necrosed brain parenchyma, removal of cerebral debris with meticulous dural closure was first stressed by Harvey Cushing, ${ }^{1}$ which laid the basic protocol for management of missile injury victims and is still followed till date. But the fear of brain abscess formation was always there and aggressive debridement was done and every attempt was made to chase and remove the lodged parenchymal fragments. But with time from World War II to Kargil War, there has been continuous improvement in management protocol of patients with craniocerebral missile injuries resulting in improved survival and better functional outcome because of the aggressive resuscitation and shift in management strategies from more to less aggressive surgery.

\section{Problems Considered}

Aggressive surgery without proper resuscitation done earlier for craniocerebral missile injuries had resulted in high mortality and poor functional outcome. Apart from poor prognostic indicators, the reason for high mortality and poor functional outcome are many viz.; poor prehospital care and delayed transport, poor or no adequate pre-op resuscitation and aggressive surgery causing increased functional brain parenchymal removal or injury. The problems are many but the two problems highlighted are decreased survival because of the inadequate pre-op resuscitation and poor functional outcome due to aggressive surgery. Therefore, the study was performed to evaluate the outcome of early and less aggressive surgical strategy and aggressive preoperative resuscitation in patients with penetrating injuries and also to look for factors prognosticating outcome.

In spite of high mortality no proper attention was given in last five decades in improving surgical techniques and resuscitation patterns of patients with craniocerebral missile injuries. It came to the limelight recently to international arena on January 8, 2011, when US Congresswoman Gabrielle Gifford was shot in the brain but recovered as timely appropriate management was done. Her successful recovery stressed the need for evidence-based treatment protocols for management of patients with gunshot wound (GSW) to the brain (Time is Brain). ${ }^{2}$ But aggressive resuscitation is considered to be one of the most important factor of survival in patients with fatal gunshot injury (46\%) improved survival as shown in the past 4 years. ${ }^{3}$

\section{Methods}

This study is a retrospective evaluation of the 96 patients admitted to Guwahati Medical College and Hospital, Guwahati, India, from January 2011 to December 2013, only high volume center in north-eastern part of India with fully equipped neurocritical care intensive care unit, 24-hour computed tomographic (CT) scan, and round the clock neurosurgery coverage. The case record and imaging of all the patients were evaluated. All the patients had complete blood investigations including prothrombin time/ international normalized ratio, and platelet count. X-ray and CT scan of head were performed. Radiological investigations were done to ascertain the amount of damage in neural tissue and bone, position of bullet with its tract, displacement of vital structures, and any evidence of secondary injury such as hematoma/edema. 3D CT reconstruction of skull were done in patients with large bone defect on local wound examination. Also, CT angiography was done in one patient with entry wound in right frontal region showing pellets and hematoma in ACA-ACOM [anterior cerebral artery - anterior 

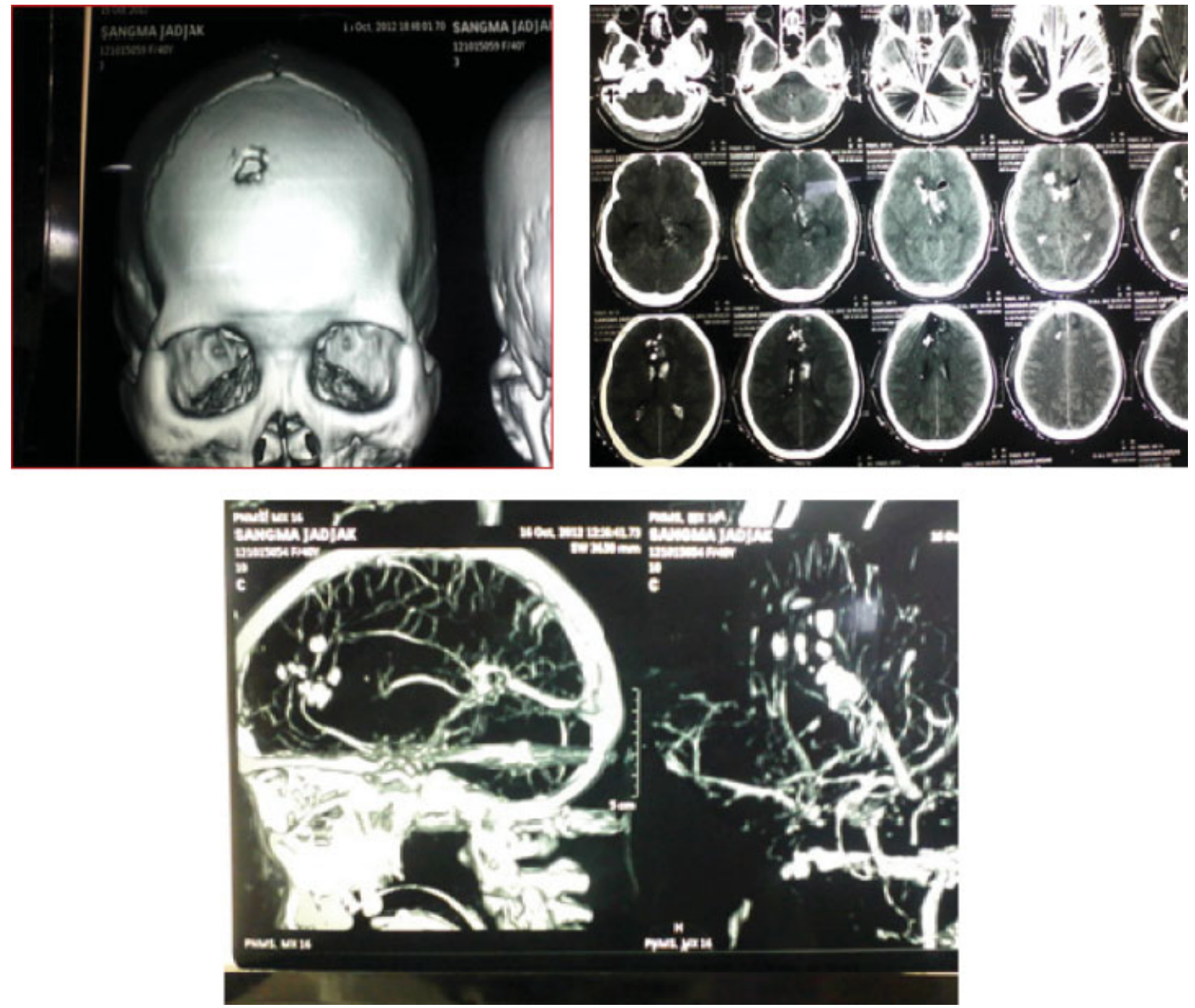

Fig. 1 Case 22. Photograph of a patient with gunshot injury in right frontal region with bullet in postfossae and hematoma in ACA-ACom junction.

communicating artery] complex to rule out traumatic aneurysm or vascular injury (as shown in -Fig. 1 but angiography was negative and did not reveal any aneurysm). Aggressive resuscitation was done in all hemodynamically unstable patients and patients with coagulopathy either with blood products, hyperosmolar therapy, or ionotropic support on protocols of damage control resuscitation. Blood product therapy consisted of packed red blood corpuscles, fresh frozen plasma (FFP), and platelets. Hypertonic saline was used for hyperosmolar resuscitation and for ionotropic support dopamine, adrenaline, and vasopressin were used. Aggressive resuscitation included early administration of blood products and hypertonic saline and restricted use of intravenous fluids. Every attempt was made to prevent hypotension once base line blood pressure (BP) was achieved. Grossly, for every one unit of packed cell transfusion one unit of FFP was given. ${ }^{4}$ The input and output charting was maintained hourly. The type and dose of vasopressors and amount of hypertonic saline solution was also noted.
Neurosurgical interventions were done if the patients have one of the following criteria:

1. Glasgow Coma Scale (GCS) scores of $>3$ but $<8$ without hypotension but with reacting pupils.

2. GCS $>8$ with intracranial bone fragments/significant clot.

However, no surgical intervention done for patient with GCS 3 , fixed dilated pupil, and patients in shock without significant intracranial hematoma.

Neurosurgical procedures contemplated on 78 of 96 patients with 16 patients having polytrauma to chest and abdomen. Overall, 10 patients with intact dura or dural injury without intracranial hematoma/mass effect needed simple wound debridement and closure and 6 patients with craniofacial injuries needed simple repair of wound. Two patients GCS $<4$ were managed conservatively. Overall, 28 patients were hemodynamically unstable on primary survey and had to be first aggressively resuscitated with blood products, hyperosmolar therapy, and inotropic and BP raised $>110 / 70$ before considering for emergency 
craniotomy. Overall, six patients needed simple wound debridement and closure. The patients were followed up at 3 and 6 months' interval.

\section{Technique of Less Aggressive Surgery}

Traditionally, debridement of missile injury tract is done with the use of suction and forceps aggressively removing suspected devitalized and necrotic brain tissue until normal looking brain tissue is seen. We have used mild suction for removing superficial necrotic tissue at the entry of the tract. Normal saline for gentle irrigation of the missile tract as debridement technique in most of the cases. Long tract was irrigated with the help of silicon catheter of external ventricular drainage kit without stellate. Diluted hydrogen peroxide in cases with grossly contaminated wound with hairs and foreign body, but it is washed completely with normal saline. After irrigation, the necrotic tissues are removed with cottonoid. Mild gentle suction (closed tip with side holes) is used in patients with small solitary tract single lobar injury which is termed as relatively less aggressive in our study. No brain dissection was done either for debridement or to remove inaccessible and deep fragments. Suction and forceps are not used at all in multitracts-multilobar injury and in deeper tracts. ${ }^{5}$

All the patients were started with broad spectrum antibiotics and antiepileptics and cerebral decongestants after securing airway, breathing, circulation, and spine. Primary goal of surgery was of wound debridement, removal of any bone fragment with or without removal of the metallic bullet. However, no attempt had been made to chase and remove bullets or bone fragments from inaccessible areas or deeper cortical areas. Scalp flap was raised including the previous inflicted wound as per requirement of the exposure. Extension of the scalp wound was avoided and considered only in a few patients. In three patients with gross skin loss dural closure, wound debridement was done as primary surgery followed by skin grafting in two patients and flap cover in one patient. Preparations were made for microscopic and vascular surgery in all cases. Relatively more aggressive surgery was done in patients with solitary tract lobar injury in noneloquent area. Fascia lata graft was used as dural substitute. Minimum debridement of the missile tract was done and wound irrigated with diluted hydrogen peroxide and normal saline and removing devitalized tissues with cottonoid was also performed. Suction was not used for debridement of deep missile tracts. Artificial dural substitute was used in single patient with small cortical injury and healthy scalp wound. Hemostasis is maintained with bipolar, gelfoam, and surgical dura closed in water tight manner with 4-0 vicryl. Craniotomy is preferred to craniectomy wherever feasible. Infected scalp wound is debrided, margins freshened, and irrigated with $1 \%$ povidone-iodine. Infected wound was closed in single interrupted sutures with nylon, whereas the raised scalp flap closed in double layer. Closed subgaleal drain was kept for 12 to 18 hours after watertight dural closure. Cultures and sensitivity tests were done in all cases from wound, and antibiotics were changed as per culture reports. Delayed cranioplasty ( $>6$ months) was done as second stage procedure with bone cement or titanium mesh fixed with miniplate and screws.

Functional outcome was assessed by Glasgow Outcome Score (GOS).

Statistical correlation of outcome variables was done using Chi-square/Fischer exact test (WHO software “EPIINFO" version 6.04D).

\section{Results}

The overall series was comprised of age group ranging from 5 to 68 years with a mean age of 26 years. There were eight patients in pediatric age group. Overall, 74 (86\%) were males and the remaining 12 (14\%) patients were females. Patients had common mode of injury from shrapnel or improvised explosive device due to terrorist attack, firearm in civilian practice either because of the alleged assault ( $n=32.74 \%)$, suicidal attempt ( $n=3.7 \%$ ), or accidental injury ( $n=8.19 \%)$. Hospital arrival time was from 2 to 28 hours ( $15 \pm 13.5$ hours); times of injury for most of the cases were from 6 PM to 3 AM (68\%) versus 3 Am to 6 PM (32\%). Missile wound entry site included occipital (41\%), frontal (20\%), temporal (23\%), and parietal (23\%). We have also encountered eight patients with missile injuries to spine and two patients with gunshot injuries (GSI) to brachial plexus. Post-op CT showed retained fragments in $42 \%$ of cases. Surgical mortality was $18 \%$ and overall mortality was $33 \%$.

Overall, 66\% ( $n=52)$ patients were admitted with an admission GCS score of less than 8. Intracranial metallic pellet was found in $86 \%$ and intracranial bone fragments found in $50 \%$ patients. One patient presented with cerebrospinal fluid (CSF) rhinorrhea because of the close range frontobasal injury and required anterior skull base repair.

Table 1 Showing clinical and radiological features

\begin{tabular}{|l|l|l|}
\hline Cranial GSW presentation & $\begin{array}{l}\text { No of } \\
\text { patients }\end{array}$ & $\begin{array}{l}\text { \% of } \\
\text { patients }\end{array}$ \\
\hline GCS $<8$ & 52 & 66 \\
\hline GCS $>8$ & 36 & 46 \\
\hline Intracranial metallic pellet & 67 & 86 \\
\hline Intracranial bone fragments & 39 & 50 \\
\hline Diffuse injuries & 22 & 27 \\
\hline Focal neurological deficits & 56 & 71 \\
\hline Contusions & 58 & 74 \\
\hline Intracranial hematoma & 36 & 46 \\
\hline Absent brain stem reflexes & 6 & 7 \\
\hline Seizures & 22 & 28 \\
\hline Systemic injury & 12 & 15 \\
\hline
\end{tabular}

Abbreviations: GCS, Glasgow Coma Scale; GSW, gunshot wound. 
Overall presentation is described in - Table 1.

Different surgical approaches were considered depending on the following three important factors (1) pattern of missile tract, (2)presence of metallic and bony fragments, and (3) hematoma with mass effect. Different surgical approaches used in our study included frontotemporal exposure $(41 \%, n=32 / 78)$, frontoparietal exposure $(22 \%$, $n=18 / 78)$, retrosigmoid exposure ( $2 \%, n=2 / 78)$, combined middle and posterior fossa approach $(2 \%, n=2 / 78)$, and decompressive craniectomy $(43 \%, n=24 / 78)$. A total of 10 patients needed retrosigmoid exposure which is quite uncommon as shown below in - Fig. 2. Procedures done were debridement of devitalized tissue (100\%, $n=78 / 78)$, extraction of bone fragment $(43 \%, n=17 / 39)$, extraction of metallic pellet $(62 \%, n=42 / 67)$, lax duraplasty $(100 \%$, $n=39 / 39)$, debridement of missile tract (100\%, $n=78 / 78)$. Six patients with GCS less than five patients were managed conservatively as they did not have any mass effect on CT scan. Overall, 10 patients had intact dura and were managed with simple wound suturing. One patient with craniofacial injury managed primarily with suturing and needed reconstruction at later date and another patient with cranio-orbital injury was managed by evisceration. Overall, 36 patients were operated within 8 hours and 22 patients were operated within 14 hours (-Table 2 ).

\section{Complications}

In regard to early postoperative complications, 44\% had wound infection, CSF leak, and meningitis in $38 \%$, chest infection in $23 \%$, seizure in $18 \%$, acute renal failure in $4 \%$, and
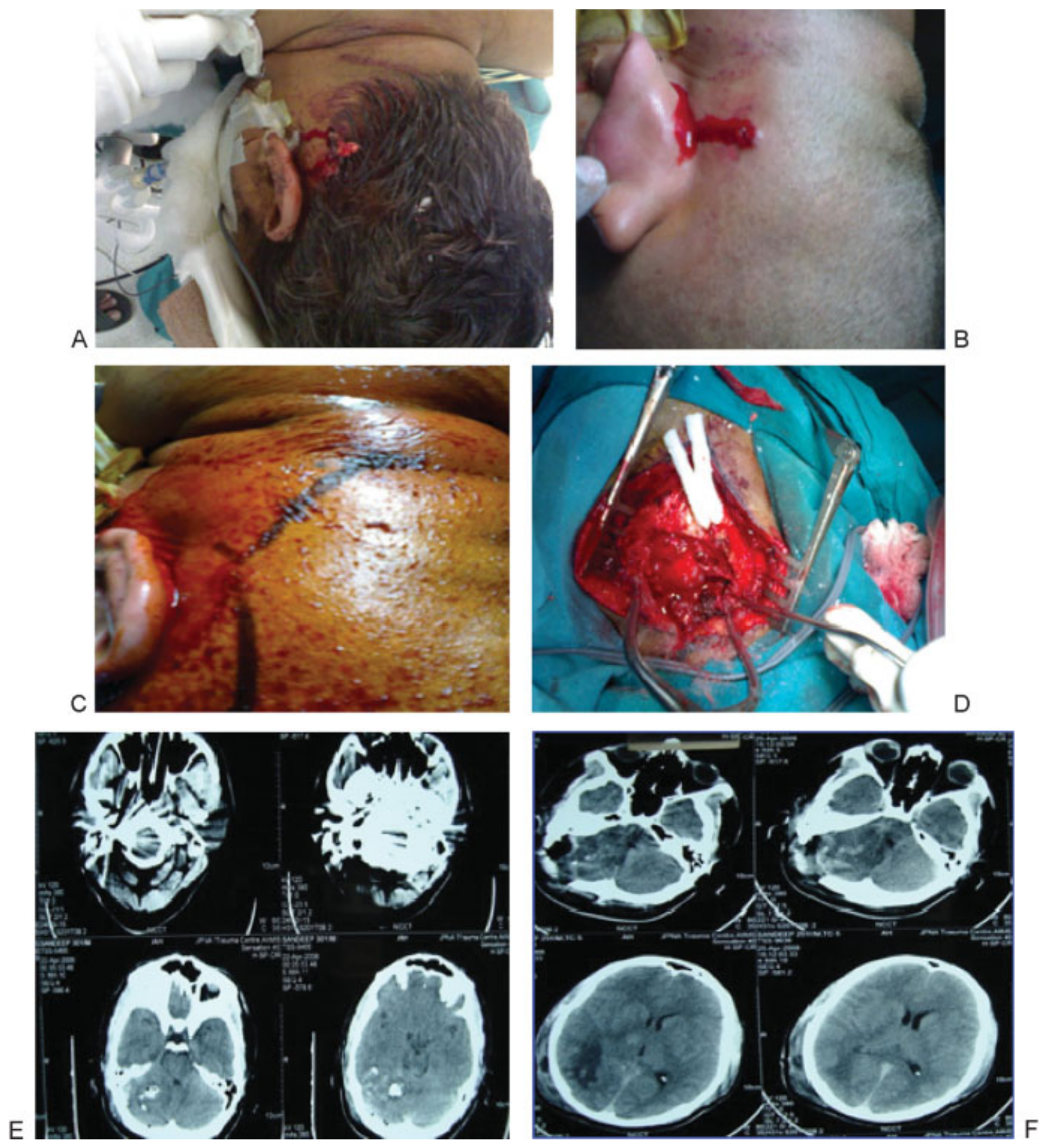

Fig. 2 Clinical Photograph. Case 11. Gunshot injury at retromastoid region, thorough debridement and removal of metallic fragments done. (A, B) Pre-op image; (C, D) Intra-op image; (E) Pre-op CT scan (F) Post-op CT scan. 
58 Paradigm Shift in Management Strategies of Craniocerebral Missile Injuries Singh et al.

Table 2 Showing surgical exposure of cranial missile injury cases

\begin{tabular}{|l|l|}
\hline Surgical exposure & \% of patients \\
\hline $\begin{array}{l}\text { Frontotemporo parietal } \\
\text { decompressive craniectomy }\end{array}$ & 43 \\
\hline Frontotemporal & 41 \\
\hline Frontoparietal & 22 \\
\hline Retrosigmoid & 2 \\
\hline Middle + postfossae & 2 \\
\hline
\end{tabular}

deep vein thrombosis in $2 \%$. Hospital stay (mean \pm 2 SD) was $12-40$ (26 \pm 14$)$ days. GOS was $14 \%$ for good recoveries, $29 \%$ were moderately disabled, $7 \%$ was severely disabled, and $21 \%$ were remained in vegetative state at 6 months' follow-up, in hospital mortality was $23 \%$. Wound infection was managed with regular dressing and appropriate antibiotics. CSF leak stopped spontaneously in almost all except one patient needed skull base repair. Seizure was controlled with single antiepileptics in 31 patients (phenytoin sodium) and 12 patients needed double antiepileptics (phenytoin sodium plus clobazam). Subcutaneous low molecular heparin was used to manage deep vein thrombosis and one patient of renal failure improved with hemodialysis. None of the patient developed brain abscess on 6-month follow-up period.

Patients who were treated surgically, with the admission GCS of 6 to 8 had statistically significant survival benefit and is described in - Table 3.

Resuscitation patterns of patients with craniocerebral missile injuries is shown in - Table 4 later.

Different clinical variables were noted and the most important of them were preoperative GCS, age, and BP. Functional outcome was assessed in all patients by Glasgow Outcome Scale. The patients with better pre-op GCS $>8$ and with hemodynamic stability had better outcome. Besides this, the patient with better GCS and stable BP had decreased hospital stay as described in - Table 5. Age is not implicated as a variable as number of cohorts older than
55 years is much less than its counterparts and is not considered to be significant clinically.

Favorable prognosticators found to be as follows:

- GCS > 8: Better neurological outcome and survival.

- GCS 6-8: Surgery offered better survival.

- Hemodynamic stability (BP > $90 \mathrm{~mm}$ systolic): Better outcome.

- Unfavorable outcomes are as follows:

- GCS $<4$.

- Patients with polytrauma in shock.

- Bilateral fixed and dilated pupils.

- Abnormal respiratory pattern.

- Absent brain stem reflexes.

- CT scan showing diffuse cerebral injuries, brain stem injury, and intraventricular bleed.

\section{Discussion}

Though there has been a worldwide increase in the incidence of gunshot injuries, still there is relatively less published series in English literature on craniocerebral and brachial plexus gunshot injuries.

GSI to head accounts for almost 35\% of deaths from brain injury in persons younger than 45 years of age.

Increased incidence of brain abscess was reported in patients with retained fragments in casualties during early part of World War II and Korean War, and it was advocated that aggressive surgery may decrease incidence of post-op brain abscess formation. ${ }^{6}$

Sherman et $\mathrm{al}^{7}$ has described the evolution of management protocol of gunshot injury from World War I to Vietnam War and has shown an overall mortality rate of $23 \%$. Early surgery and organized evacuation of GSW victims was advocated by them from the results of Korean War. Most common complication in their study was CSF fistula followed by seizure and meningitis.

Studies on victims of Vietnam War also showed aggressive surgery decreased the infection rate from 53 to $15 \%$ and surgical mortality. But the phase I and phase II studies failed to establish any correlation between retained fragments and incidence of brain abscess formation..$^{8,9}$

Table 3 Showing admission GCS score and mode of treatment

\begin{tabular}{|l|l|l|l|l|}
\hline Mode of treatment & Admission GCS score & Survived & Died & $p$ Value \\
\hline \multirow{5}{*}{ Surgery } & $3-5$ & None & 2 & - \\
\cline { 2 - 5 } & $6-8$ & 40 & 10 & $<0.05$ \\
\cline { 2 - 5 } & $9-12$ & 17 & 1 & $<0.05$ \\
\cline { 2 - 5 } & $13-15$ & 8 & 0 & NS \\
\hline \multirow{5}{*}{ Conservative } & $3-5$ & 0 & 5 & 1 \\
\cline { 2 - 5 } & $6-8$ & 0 & 0 & - \\
\cline { 2 - 5 } & $9-12$ & 2 & 0 & HC mortality \\
\cline { 2 - 5 } & $13-15$ & 10 & & - \\
\hline
\end{tabular}


Table 4 Resuscitation

\begin{tabular}{|l|l|l|l|}
\hline Aggressive resuscitation & Survived \% & Died \% & $\boldsymbol{p}$ Value \\
\hline Blood products & 55 & 30 & $<0.05$ \\
\hline Hypertonic saline & 51 & 26 & $<0.05$ \\
\hline lonotropic & 39 & 29 & $<0.5$ \\
\hline
\end{tabular}

In the series reported by Kaufman et $\mathrm{al}^{10}$ only $5 \%$ of 48 survivors were vegetative or severely disabled, $29 \%$ experienced a moderate disability, and $67 \%$ experienced a good outcome. Only 38\% remained in the hospital for either rehabilitation or lengthy acute care treatment. Selden et $\mathrm{al}^{11}$ also reported a good outcome in those patients who survived a suicide attempt following gunshot injury, with 91\% (10/11) experiencing a good outcome. But the sample size is too small to draw any conclusion.

A conservative approach was reported by Brandvold et $\mathrm{al}^{12}$ in their experiences in Israeli soldiers injured in Lebanon conflict between 1982 and 1985. The less aggressive approach with emphasis on preservation of cortical tissue with conscious effort not to search or remove in driven fragments resulted in zero suppurative complications.

Levy $^{13}$ from Department of Neurological Surgery, University of Southern California School of Medicine, Los Angeles, CA, found a significant relationship between operative intervention and survival $(p \leq 0.01)$ in patients with admission GCS scores of 6 to 8 . No significant relationships between operative intervention and survival were found in patients with admission GCS scores of 9 to 12 and 13 to 15 . A significant relationship between operative intervention and morbidity $(p \leq 0.01)$ was also demonstrated in patients with an admission GCS score of 12 to 15 . No significant relationships between operative intervention and morbidity were found in patients with an admission GCS score of 6 to 8 and 9 to 12 .
But it was Clark et al ${ }^{14}$ who concluded that patients with a GCS of 3 on presentation invariably die, regardless of aggressive fluid or surgical management.

Kennedy et al have shown that CT scan findings help predict survival in patients with GLS. ${ }^{5-13}$ The remainder made a good outcome (mild or no disability).

Grahm et al $^{16}$ have shown brain stem injury to be worst prognostic criteria and did not consider them to be surgical candidates. GCS has been shown to be most important prognostic factors in most of the studies ${ }^{11,13,17}$ as found in our studies too. We have not operated patients with GCS less than 4 and with fixed and dilated pupils. Shaffrey et al ${ }^{18}$ reported a mortality rate of $91.4 \%$ with a GCS score of 3 to 4 , and $61.2 \%$ with a GCS score of 5 to 8 , in patients with gunshot injuries to the brain. Overall, 66\% patients with GCS $<8$ had poor functional outcome in our study which is comparable with that of Shaffrey et al. ${ }^{13}$

Intraventricular bleed is a poor prognostic indicator as suggested by Singh et $\mathrm{al}^{5}$ as depicted in our study. Besides this, they have shown the benefits of less aggressive debridement in missile injury victims of Kargil War. Vrankovic et al $^{19}$ also showed the benefits of less aggressive surgery by managing patients during Croatian conflict by gentle irrigation of necrotic tissue and removal of bone fragments that presented during surgery. Both the series showed that there was no increase in mortality rates or intracranial sepsis.

Tsuei et $\mathrm{al}^{20}$ has showed in their study that the most important prognostic criteria were GCS and CT scan as indicated in our study also and has shown overall mortality rate of $31.3 \%$. Bhat et $\mathrm{al}^{21}$ et al has shown surgical mortality of $36.4 \%$ with 28 years' study of craniocerebral missile injuries in Banaras Hindu University, India. Singh et $\mathrm{al}^{5}$ has shown a surgical mortality of $8.5 \%$ from less aggressive surgery following victims of Kargil War. Although the surgical mortality rate from aggressive surgery from Vietnam War has shown to be $11 \%$. The surgical mortality of our study was $12.8 \%$, which very well matches with the result of study of Hammon. ${ }^{22}$ The high overall mortality rate of $33 \%$ of our study is because of the fact that we have more cases of patients with gunshot injury and have

Table 5 Showing the different clinical variables, GOS, and hospital stay of different cohorts

\begin{tabular}{|c|c|c|c|c|}
\hline Clinical variable & $\begin{array}{l}\text { Functional outcome } \\
\text { favorable GOS }\end{array}$ & $\begin{array}{l}\text { Functional outcome } \\
\text { unfavorable GOS }\end{array}$ & p Value & Hospital stay (d) \\
\hline \multicolumn{5}{|l|}{ GCS } \\
\hline GCS $>8(n=52)$ & $80 \%$ & $20 \%$ & & $12-18$ \\
\hline GCS $<8(n=26)$ & $44 \%$ & $66 \%$ & $<0.05$ & $21-40$ \\
\hline \multicolumn{5}{|l|}{ BP } \\
\hline Normal, $n=50$ & 42 & 8 & & $12-14$ \\
\hline Hуро, $n=28$ & 11 & 17 & $<0.01$ & $21-40$ \\
\hline \multicolumn{5}{|l|}{ Age group } \\
\hline$<55$ y & $29 \%$ & $35 \%$ & & $12-38$ \\
\hline$>55 y$ & $14 \%$ & $22 \%$ & & $12-40$ \\
\hline
\end{tabular}

Abbreviations: BP, blood pressure; GCS, Glasgow Coma Scale; GOS, Glasgow Outcome Score. 
included polytrauma victims and because of the delay in shifting the patients due to difficult terrain and remote areas to our institute.

Solmaz et $\mathrm{al}^{17}$ concluded that early surgery and short transportation time improves outcome in management of such victims of gunshot injury. The casualties if transported early has better chances of survival as we have shown in our study.

No attempt was made to chase and remove fragments situated deep inside as the retained fragments were not found to increase the seizure incidence as reported by Weiss et al. ${ }^{23}$

It was DuBose et $a{ }^{3}{ }^{3}$ who in their study reported an improved survival rates in patients with severe traumatic brain injury who were treated with $5 \%$ hypertonic saline. Hypertonic saline recruits fluid in intravascular space and stabilizes BP and also decreases intracranial pressure besides improving neuronal survival.

Joseph et $\mathrm{al}^{4}$ have demonstrated in their landmark study that survival rate increased incrementally over time to a rate of $46 \%$ with early aggressive resuscitation of all patients with GSWs to the brain regardless of their presenting clinical picture.

\section{Conclusion}

Craniocerebral missile injuries are challenging problems for the neurosurgeons and incidence of firearm injuries to head are increasing with the increase of civilian violence, easy availability of automatic guns, and terrorism and are very fatal. Improvement in prehospital care and rapid evacuation has provided more time to such victims so that they can be transferred to a higher center. 3D CT head helps in better preoperative planning and designing the incision. BP must be raised before emergency craniotomy with aggressive resuscitation. Early and appropriate microsurgical procedures, wound debridement and water-tight duraplasty are same and still the mainstay of treatment. Early surgical candidates has less chances of development of meningitis and wound infection and antibiotics are not alternative to early surgery. Bone must be preserved as much as possible and replaced immediately in victims where there is no gross contamination. Late cranioplasty is preferable as osteomyelitis may have delayed presentation. Postresuscitation GCS, hemodynamic stability, and CT scan findings prognosticated outcome. Overall, $80 \%$ of patients with GCS $>8$ had better functional outcome. Correction of coagulopathy improves survival. Retained bony fragments are not epileptogenic. Mortality and hospital stay are more in hemodynamically unstable patients in patients with poor GCS. But over the years, there has been decline in mortality and morbidity following changing trends of management from more to less aggressive surgical approach and adequate preoperative resuscitation. The better functional outcome may be because of the less cortical damage due to less aggressive surgery combined with aggressive resuscitation. Surgical mortality rates are acceptable and no increased incidence of brain abscess was found due to less aggressive surgery. We recommend that all patients who are referred to higher center in unstable condition must be adequately resuscitated during transportation and surgery for improved survival and better outcome.

\section{Conflict of Interest}

The authors have no conflict of interest and do not promote any products or medicine.

\section{References}

1 Cushing $\mathrm{H}$. A study of series of wounds involving the brain and its enveloping structures. Br J Surg 1918;5:558-684

2 Lin DJ, Lam FC, Siracuse JJ, Thomas A, Kasper EM. "Time is brain" the Gifford factor - or: Why do some civilian gunshot wounds to the head do unexpectedly well? A case series with outcomes analysis and a management guide. Surg Neurol Int 2012;3:98

3 DuBose JJ, Barmparas G, Inaba K, et al. Isolated severe traumatic brain injuries sustained during combat operations: demographics, mortality outcomes, and lessons to be learned from contrasts to civilian counterparts. J Trauma 2011;70(1): 11-16, discussion 16-18

4 Joseph B, Aziz H, Pandit V, et al. Improving survival rates after civilian gunshot wounds to the brain. J Am Coll Surg 2014; 218(1):58-65

5 Singh P, Misra GS, Singh A, Murthy MGK. Missile injuries of brainan experience in northern sector. Medical J Armed Forces India 2003;59(4):290-297

6 Wannamaker GT, Pulaski EJ. Pyogenic neurosurgical infections in Korean battle casualties. J Neurosurg 1958;15(5):512-518

7 Sherman WD, Apuzzo MLJ, Heiden JS, Petersons VT, Weiss MH. Gunshot wounds to the brain-a civilian experience. West J Med 1980;132(2):99-105

8 Rish BL, Caveness WF, Dillon JD, Kistler JP, Mohr JP, Weiss GH. Analysis of brain abscess after penetrating craniocerebral injuries in Vietnam. Neurosurgery 1981;9(5):535-541

9 Myers PW, Brophy J, Salazar AM, Jonas B. Retained bone fragments after penetrating brain wounds. Long term follow up in Vietnam veterans. J Neurosurg 1989;70:319A

10 Kaufman HH, Makela ME, Lee KF, Haid RW Jr, Gildenberg PL. Gunshot wounds to the head: a perspective. Neurosurgery 1986; 18(6):689-695

11 Selden BS, Goodman JM, Cordell W, Rodman GH Jr, Schnitzer PG. Outcome of self-inflicted gunshot wounds of the brain. Ann Emerg Med 1988;17(3):247-253

12 Brandvold B, Levi L, Feinsod M, George ED. Penetrating craniocerebral injuries in the Israeli involvement in the Lebanese conflict, 1982-1985. Analysis of a less aggressive surgical approach. J Neurosurg 1990;72(1):15-21

13 Levy ML. Outcome prediction following penetrating craniocerebral injury in a civilian population: aggressive surgical management in patients with admission Glasgow Coma Scale scores of 6 to 15. Neurosurg Focus 2000;8(1):e2

14 Clark WC, Muhlbauer MS, Watridge CB, Ray MW. Analysis of 76 civilian craniocerebral gunshot wounds. J Neurosurg 1986;65(1):9-14

15 Kennedy F, Gonzalez P, Dang C, Fleming A, Sterling-Scott R. The Glasgow Coma Scale and prognosis in gunshot wounds to the brain. J Trauma 1990;1993(35):75-77

16 Grahm TW, Williams FC Jr, Harrington T, Spetzler RF. Civilian gunshot wounds to the head: a prospective study. Neurosurgery 1990;27(5):696-700, discussion 700

17 Solmaz I, Kural C, Temiz C, et al. Traumatic brain injury due to gunshot wounds: a single institution's experience with 442 consecutive patients. Turk Neurosurg 2009;19(3):216-223

18 Shaffrey ME, Polin RS, Phillips CD, Germanson T, Shaffrey CI, Jane JA. Classification of civilian craniocerebral gunshot wounds: a multivariate analysis predictive of mortality. J Neurotrauma 1992;9(Suppl 1):S279-S285

19 Vrankovic D, Splavski B, Hecimovic I, Glavina K, Dmitrovic B, Mursic B. Analysis of 127 war inflicted missile brain injuries sustained in north-eastern Croatia. J Neurosurg Sci 1996;40(2):107-114 
20 Tsuei YS, Sun MH, Lee HD, et al. Civilian gunshot wounds to the brain. J Chin Med Assoc 2005;68(3):126-130

21 Bhat AR, Mohanty S, Sharma V, Agrawal R. Cranio-cerebralmissile injuries, an experience of 28 years at BHU. Neurosurgery Quarterly Journal of Surgical Sciences 1998;34:19-23
22 Hammon WM. Analysis of 2187 consecutive penetrating wounds of the brain from Vietnam. J Neurosurg 1971;34(2 Pt 1):127-131

23 Weiss GH, Feeney DM, Caveness WF, et al. Prognostic factors for the occurrence of posttraumatic epilepsy. Arch Neurol 1983; 40(1):7-10 